Practitioners working in Australian mental health services are faced with the challenge of providing appropriate evidence-based interventions that lead to measurable improvement and good outcomes. Current government policy is committed to the development of strategic mental health research. One focus has been on under-researched practice areas, which include the development of psychosocial rehabilitation systems and models that facilitate recovery.

To meet this challenge, an Australian rehabilitation service formed a collaborative partnership with a university. The purposes of the collaboration were to implement new forms of service delivery based on consumer need and evidence and to design research projects to evaluate components of the rehabilitation programme. This article examines the process of developing the collaboration and provides examples of how research projects have been used to inform practice and improve the effectiveness of service delivery. Challenges to the sustainability of this kind of collaboration are considered.

\title{
Occupational Therapy and Clinical Research in Mental Health Rehabilitation
}

\author{
Chris Lloyd, Robert King and Hazel Bassett
}

\section{Introduction}

In Australia, a number of key policy documents under the National Mental Health Strategy are setting the research agenda (Australian Health Ministers 1992, 1998, 2003,

Commonwealth Department of Health and Family Services 1997, Jorm et al 2002). These documents emphasise the use of guidelines, research evidence and clinical outcome studies to determine appropriate interventions, establish benchmarks and identify best models of practice, and routinely monitor the effectiveness of service delivery and clinical practice. Mental health professionals are required systematically to monitor and evaluate their clinical practice, consistent with the policy documents, to ensure the best possible outcomes for consumers, family members and/or carers.

According to NSW (New South Wales) Health (2002), best practices in mental health rehabilitation are changing in response to new knowledge from research, clinical practice and consumer and carer input. It has been suggested that there is a strong case for establishing evidence-based and cost-effective programmes to improve the quality of mental health rehabilitation (NSW Health 2002). Therein lies the challenge for occupational therapists working in mental health rehabilitation. Occupational therapists need to consider the use of economic evaluation to meet the overall objective of reducing disability through the utilisation of effective rehabilitation strategies designed to improve efficiency in service delivery.
This article explores the importance of using research to inform practice for occupational therapists working in mental health rehabilitation. Practical suggestions to overcome the research-practice gap are then considered, followed by an example of a research collaboration that was developed between a regional mental health rehabilitation team and a metropolitan university.

\section{Literature review}

\section{Research in practice}

The use of appropriate research evidence in mental health is a priority under current Australian policies (Australian Health Ministers 2003) and mirrors the policy directions that have taken place in the United Kingdom (Department of Health 1997). Although the occupational therapy literature has urged occupational therapists to collaborate with researchers (DePoy and Gallagher 1990, Pranger and Brown 1990, Cusick 1994), there exists a low level of research involvement by occupational therapy practitioners (Waine et al 1997, Cusick 2000, 2001). A number of factors have been identified concerning the reasons that research has not been a high priority for occupational therapy clinicians. These include:

- Time not being allowed for research (Waine et al 1997)

- Lack of financial support (Waine et al 1997)

- Low priority by administrators (Majnemer et al 2001) 
- Lack of occupational therapist researcher (or mentor) on site (Majnemer et al 2001)

- Lack of collaboration between academic institutions and the clinical field (Cusick 1994).

\section{Research policies}

With continued reform of mental health services, occupational therapists are no longer able to view research as being a low priority for them. One of the key priority themes of the Australian research agenda is to foster research, innovation and sustainability (Australian Health Ministers 2003). Strengthening quality is seen as being important, with the notion of quality emphasising appropriate evidence-based care that leads to measurable improvement and good outcomes. Psychiatric disability has not only substantial implications for the individual and his or her family but also economic costs (Sane Australia 2002). Current policies demand that, in an environment of limited resources and increasing demand, it is important to justify resource usage in terms of consumer outcomes and returns on investments (Australian Health Ministers 2003). It is acknowledged that research in this area is in its infancy in Australia and that it is necessary to form partnerships in order to promote research that informs the relevant stakeholders strategically. The development of a strong research culture within the mental health sector is seen as being crucial (Australian Health Ministers 2003).

\section{Strategies}

Adopting research within the clinical setting is a very real challenge for occupational therapists. This requires occupational therapists to change their customary work behaviours so as to become receptive to change and to make a commitment to the process of change. Whiteley (1995, p34) described change 'as the negotiation or the renegotiation of shared meaning about what is to be valued, believed in and aimed for'. According to Brown and Rodger (1999), the need to define strategies to facilitate the adoption of research in practice has become pressing for the profession. A key strategy is to engage in continuing empirical evaluation of practice.

This strategy involves undertaking a continuing empirical evaluation of clinical practice. The 'scientist-practitioner' model (Corrie and Callahan 2000) was developed within the allied health profession of psychology. This approach has not been a standard feature of occupational therapy practice. Occupational therapists have had limited participation in clinical research (Cusick 2001). For example, an Australian study conducted by Lloyd (2003) found that mental health occupational therapists reported spending less that $4 \%$ of their work time in activities involving research and evaluation.

Collaboration has the potential to overcome the low level of involvement in clinical research. Collaborative research is an important strategy for change (Cusick 1994). Harrison and Eaton (1999) considered that using routine outcome measures was the key to making the shift from the research world to the real world. Routine evaluation needs to be built into usual practice activities. Evaluation alone is not research per se. Ethical approval will need to be sought if the results are to be published. The usual setting of clinical practice provides a fertile environment in which to conduct research relevant to everyday practice (Pas Colborn 1993). This paper briefly goes through the aspects of developing research collaboration that the authors see as being important, drawing on their experience of a collaboration developed between the Integrated Mental Health Rehabilitation Service, Gold Coast Hospital, and the School of Medicine, University of Queensland.

\section{Steps in collaboration between clinicians and academic researchers}

\section{Identifying resources and need for upskilling}

A first necessary step is for clinicians to identify whether they have the resources available to prepare for and conduct research. This includes dedicated time for research-related activities, library resources, the availability of information technology and access to the internet. Once this is established, clinicians need to identify their level of knowledge and skill related to clinical research and in conducting research-related activities (Alsop 1997). They require knowledge of ethical requirements in conducting research, research methodology and understanding of statistical analyses. This will then enable clinicians to incorporate evidence into practice and to develop a research programme.

\section{Setting the research agenda}

In mental health, a number of research trends have become evident. These include evaluation of service models, including psychosocial rehabilitation systems and models that facilitate recovery; access and pathways of care; outcome measurement; consumer and carer participation; and human resources, including workforce supply, organisation and environment, and training needs (Jorm et al 2002, Anthony et al 2003, Australian Health Ministers 2003).

Practice evaluation is a vital component of clinical governance and in ensuring effectiveness of service delivery. Clinicians need to develop an awareness of current research trends since it is imperative that they address pertinent and practical clinical issues that are in keeping with both policy directives and research findings (Taylor and Mitchell 1990). For research in the clinical field to be conducted successfully, the research must be clinically relevant to the everyday work of the clinicians; otherwise, it will only be seen as an imposition on their workload.

\section{Networking and partnerships}

The development of partnerships is crucial in research. Research does not happen in isolation and, to be effective in conducting 
research, it is important to determine the relevant stakeholders (Pas Colborn 1993). Identifying the relevant stakeholders and involving them in the research makes it more likely that the research will be successful (DePoy and Gallagher 1990).

First, it is necessary to identify clinical colleagues who have similar interests. The authors have found it best to establish small research teams of clinicians who share similar questions about therapy interventions to work on a project. This type of approach was designed to address such issues as staff turnover, limited staff time to devote to a project and the need for peer support. Secondly, it is necessary to determine the feasibility of partnerships outside the clinical work environment. These may include partnerships with other health districts, non-government organisations and academic institutions. The establishment of a reference group, for example experts in the field, may also provide expert information during the course of the project, such as advice on development of data collection mechanisms. It is particularly valuable to include consumer and carer participation in the reference group to ensure that interventions are kept user friendly (Trivedi and Wykes 2002).

Another useful strategy is through fostering a culture of formal research in the workplace. This may be by encouraging staff to develop the evaluation of programmes into research degree studies and/or by establishing honours research projects within the clinical setting. In this type of arrangement, the university provides primary supervision of the project and the clinician is involved in the conducting of the research and in being an associate supervisor.

\section{Mentorship and research supervision}

One of the key aspects of developing a research programme is to identify people who can act as mentors and provide research supervision. This is important since the majority of clinicians lack a background in service development, research and evaluation, funding and grant applications, ethics applications, and writing conference papers and journal articles (Majnemer et al 2001). In many cases, the mentor might be outside the clinical environment in which the clinicians are working.

Finding a mentor may involve getting in touch directly with the university to explore the research skills and interests of academic researchers; alternatively, the areas of research interests of faculty members are usually listed on the university website. Another strategy of finding a mentor may involve approaching someone whom you have heard deliver a conference paper on the topic that you are interested in researching. Yet another method is to hold a research meeting between academics and clinicians at occupational therapy conferences. This has been found to be a useful strategy in finding additional sites to be involved in the data collection of existing projects.

Research projects require continuing supervision. Supervision assists in:

- Asking the critical questions (research question) and in the setting of the research initiative

- Consideration as to the most effective means of gathering information (methodology) to answer the critical questions
- Providing focus on what action (method) will be taken to achieve the agreed methodology

- Trouble-shooting during the data collection phase

- Assisting in the interpretation of data.

\section{Roles and responsibilities}

There needs to be discussion about the roles and responsibilities of the various research team members. This is very important in order to avoid any misunderstandings, which might impede the research process (McCluskey 2003). Some considerations include identifying:

- The clinicians and academic researchers who will make up the research team

- Who will be responsible for clarifying the research question and with preparing the ethics application

- Who will be responsible for selecting the research design and method

- Who will be responsible for database literature searchers

- Which members of the team will assume primary responsibility for data collection

- Who will be responsible for data entry

- What type of arrangement needs to be made for data analysis

- The level of research supervision required

- Who will take primary responsibility for the dissemination of results, particularly for conference presentations and publication

- What processes need to be established to use the research findings in practice.

\section{Outcomes}

What is noticeable about the literature is that few of the published reports go on to demonstrate the outcomes from the proposed strategies. There is a focus on the issues surrounding why occupational therapists are slow to engage in clinical research and strategies for fostering research, notably collaborative partnerships. There are examples of publications resulting from student research projects and universities or one-off examples of joint ventures, but few demonstrate the sustainability and outcomes of the collaboration. The present collaboration has resulted in a number of research projects being conducted using a range of research methodologies, which are outlined in Table 1.

\section{Reflections on sustainability}

Although the experience reported here affirms the feasibility of establishing an effective collaboration between a service environment and a research institution, the sustainability of this kind of collaboration is as important as its establishment. Is it possible to create a lasting research collaboration in an ordinary clinical practice setting or do such collaborations arise only in the context of a temporary confluence of individuals and circumstances? 
Table 1. Examples of collaborative research projects

\begin{tabular}{|c|c|c|c|}
\hline Study & Method & Participants & Outcome \\
\hline $\begin{array}{l}\text { Leisure satisfaction } \\
\text { (Lloyd et al 2001a) }\end{array}$ & $\begin{array}{l}\text { Quantitative, cross-sectional } \\
\text { survey design }\end{array}$ & $\begin{array}{l}\text { One hundred adults with serious mental } \\
\text { illness living in the community }\end{array}$ & $\begin{array}{l}\text { Provision of a range of leisure-based } \\
\text { programmes }\end{array}$ \\
\hline $\begin{array}{l}\text { Psychiatric terminology } \\
\text { (Lloyd et al 2001b) }\end{array}$ & $\begin{array}{l}\text { Quantitative, cross-sectional } \\
\text { survey design }\end{array}$ & $\begin{array}{l}\text { One hundred and twenty-five adults with } \\
\text { serious mental illness, inpatient and outpatient }\end{array}$ & $\begin{array}{l}\text { Raised awareness of terminology to use } \\
\text { in different contexts }\end{array}$ \\
\hline $\begin{array}{l}\text { Nutrition } \\
\text { (Bassett et al 2003) }\end{array}$ & $\begin{array}{l}\text { Combined, qualitative and } \\
\text { quantitative; pilot study }\end{array}$ & Six mothers with a mental illness & $\begin{array}{l}\text { Continued use of nutrition-based } \\
\text { programmes; widened scope to include } \\
\text { other people with mental illness }\end{array}$ \\
\hline $\begin{array}{l}\text { Family psychoeducation } \\
\text { (De Groot et al 2003) }\end{array}$ & $\begin{array}{l}\text { Audit with matched controls } \\
\text { and telephone survey }\end{array}$ & $\begin{array}{l}\text { Families attended a family psychoeducation } \\
\text { programme matched with those who did not; } \\
\text { telephone interviews with both sets of families }\end{array}$ & $\begin{array}{l}\text { Redesigned the programme; currently } \\
\text { investigating effectiveness of different } \\
\text { forms of family psychoeducation provision }\end{array}$ \\
\hline $\begin{array}{l}\text { Social functioning } \\
\text { (Lloyd et al 2004) }\end{array}$ & Qualitative, focus group & Six young males with a psychotic disorder & Used to inform programme design \\
\hline $\begin{array}{l}\text { Sexual health } \\
\text { (Lloyd et al 2005) }\end{array}$ & $\begin{array}{l}\text { Quantitative, pre-test/post-test } \\
\text { design }\end{array}$ & $\begin{array}{l}\text { Twenty-four adults with mental illness, } \\
\text { acute inpatient }\end{array}$ & Continued use of the programme \\
\hline
\end{tabular}

On reflecting about the process of organising and establishing this collaboration, a number of points come to mind. The manager of the rehabilitation service had a keen interest in practice research and, since she was involved in doctoral studies, she approached one of her advisers about the prospect of becoming involved in a research collaboration. Most of the challenges involved in establishing this collaboration revolved around the clinical setting. Notably, this included heavy work schedules and persuading clinicians that there were tangible benefits to be gained from participating in research activities. In other words, this kind of collaboration is not cost neutral and there will always be a question as to who should bear the cost.

Hospitals and other service environments profess a commitment to research, but the allocation of specific resources to support such activity is less common. Research is tolerated so long as it does not compromise service provision and other core activities. This means that research has to be conducted with a high level of efficiency and it needs to take place in a clinical setting that operates with high efficiency. In practice, this means being very clear on the research process, selecting suitable measures, seeking ethical clearance early in the process and building in sufficient time for staff to attend to such processes as data entry.

It is suggested to other people wishing to establish a research collaboration that a number of elements need to be organised; for example, time management, scheduling of research supervision visits, regular communication, preparing ethics applications if there is the likelihood that the material may be publishable and being proactive in searching for sources of funding. Establishing the collaboration is not the most difficult part. The real challenge lies in keeping the momentum going especially with staff turnover and changing work schedules to accommodate staff skills and interests.

\section{Conclusion}

The collaboration described in this article has fostered the development of a research culture with a mental health rehabilitation service. There have been tangible benefits from this collaboration, including practitioners' ability to conduct research projects successfully, an improved service delivery and the dissemination of results through both presentations and publications

Service users, clinical services and universities have a clear stake in collaborative research and evaluation. The service users have benefited by improved clinical services being offered to them. They have been able to contribute actively to the research process in terms of valued feedback with regard to the structure and content of the services designed to assist them with independent community living. Clinical services depend on research to justify treatment and demonstrate the effectiveness of interventions as well as to support professional credibility. Universities need clinical settings where the actual effectiveness of clinical interventions or service development models can be tested. Such settings not only enable model testing but also contribute to the development of instruments, to the further development of theory and to increased clinical practice effectiveness.

\section{Acknowledgement}

This article is based on a conference presentation at the Mental Health Services Conference of Australia and New Zealand 2003, Canberra.

\section{References}

Alsop A (1997) Evidence-based practice and continuing professional development. British Journal of Occupational Therapy, 60(11), 503-508.

Anthony W, Rogers E, Farkas M (2003) Research on evidence-based practices: future directions in an era of recovery. Community Mental Health Journal, 39, 101-14.

Australian Health Ministers (1992) National mental health policy. Canberra: Australian Government Publishing Service. 
Australian Health Ministers (1998) Second national mental health plan. Canberra: Commonwealth Department of Health and Family Services.

Australian Health Ministers (2003) National mental health plan 2003-2008. Canberra: Australian Government.

Bassett H, Lloyd C, King R (2003) Food Cent\$: educating mothers with a mental illness about nutrition. British Journal of Occupational Therapy, 66(8), 369-75.

Brown G, Rodger S (1999) Research utilisation models: frameworks for implementing evidence-based occupational therapy. Occupational Therapy International, 6, 1-23.

Commonwealth Department of Health and Family Services (1997) National standards for mental health services. Canberra: Commonwealth of Australia.

Corrie S, Callahan M (2000) A review of the scientist-practitioner model: reflections on its potential contribution to counselling psychology within the context of current health care trends. British Journal of Medical Psychology, 73, 413-27.

Cusick A (1994) Collaborative research: rhetoric or reality? Australian Occupational Therapy Journal, 41, 49-54.

Cusick A (2000) Practitioner-researchers in occupational therapy. Australian Occupational Therapy Journal, 47, 11-27.

Cusick A (2001) The experience of clinician-researchers. American Journal of Occupational Therapy, 55, 9-18.

De Groot L, Lloyd C, King R (2003) An evaluation of a family psychoeducation programme in community mental health. Psychiatric Rehabilitation Journal, 27, 18-23.

Department of Health (1997) The new NHS: modern, dependable. $\mathrm{Cm}$ 3807. London: Stationery Office.

DePoy E, Gallagher C (1990) Steps in collaborative research between clinicians and faculty. American Journal of Occupational Therapy, 44, 55-59.

Harrison G, Eaton W (1999) From research world to real world: routine outcome measures are the key. Current Opinion in Psychiatry, 12, 187-89.

Jorm A, Griffiths K, Christensen H, Medway J (2002) Research priorities in mental health. Canberra: Centre for Mental Health Research, Australian National University, Commonwealth of Australia.

Lloyd C (2003) Occupational therapy practice in restructuring mental health services. Unpublished doctoral dissertation. St Lucia: University of Queensland.

Lloyd C, King R, Lampe J, McDougall S (2001a) The leisure satisfaction of people with psychiatric disabilities. Psychiatric Rehabilitation Journal, 25, 107-13.

Lloyd C, King R, Bassett H, Sandland S, Savige G (2001b) Patient, client or consumer? A survey of preferred terms. Australasian Psychiatry, 9, 321-24.
Lloyd C, Williams PL, Sullivan D (2004) Kick'n'On: helping young males kick back into life. Australian e-Journal for the Advancement of Mental Health, 3(2). Available at: www.auseinet.com/journal/vo/3iss2/lloyd.pdf Accessed on 2.11.04.

Lloyd C, Williams PL, King R (2005) A sexual health programme implemented in a psychiatric inpatient unit. New Zealand Journal of Occupational Therapy, 52(1), 26-32.

Majnemer A, Desrosiers J, Gauthier J, Dutil E, Robichaud L, Rousseau J, Hebert L (2001) Involvement of occupational therapy departments in research: a provincial survey. Canadian Journal of Occupational Therapy, 68, 272-79.

McCluskey A (2003) Occupational therapists report a low level of knowledge, skills and involvement in evidence-based practice. Australian Occupational Therapy Journal, 50, 3-12.

NSW Health (2002) Framework for rehabilitation for mental health. Sydney: NSW Health Department.

Pas Colborn A (1993) Combining practice and research. American Journal of Occupational Therapy, 47, 693-703.

Pranger T, Brown G (1990) Collaborative research: campus and clinic working together. Canadian Journal of Occupational Therapy, 57, 268-72.

Sane Australia (2002) Schizophrenia: costs - an analysis of the burden of schizophrenia and related suicide in Australia. Melbourne: Sane Australia.

Taylor E, Mitchell M (1990) Research activities of occupational therapy clinicians. American Journal of Occupational Therapy, 44, 350-55.

Trivedi P, Wykes T (2002) From passive subjects to equal partners: qualitative review of user involvement in research. British Journal of Psychiatry, 181, 468-72.

Waine M, Magill-Evans J, Pain K (1997) Alberta occupational therapists' perspectives on and participation in research. Canadian Journal of Occupational Therapy, 64, 82-88.

Whiteley A (1995) Managing change: a core values approach. Melbourne: Macmillan Education Australia.

\section{Authors}

Chris Lloyd, PhD, Senior Lecturer, Division of Occupational Therapy, University of Queensland, St Lucia, Q 4072, Australia.

Email: c.lloyd@shrs.uq.edu.au

Robert King, PhD, Senior Lecturer, Department of Psychiatry, University of Queensland, and The School of Mental Health, The Park Hospital, Queensland, Australia.

Hazel Bassett, BOccThy, MMedSc, Senior Occupational Therapist, Rehabilitation Services, Gold Coast Hospital, Queensland, Australia. 